\title{
GENERATING VISUAL MENTAL IMAGES: DEFICIT AFTER BRAIN DAMAGE
}

\author{
CARLA STANGALINO,* CARLO SEMENZA $\ddagger$ and SARA MONDINI $\dagger$ \\ *Clinica Neurologica III Università di Milano, Italy; and †Dipartimento di Psicologia Generale, \\ Università di Padova, Italy
}

(Received 21 August 1994; accepted 6 February 1995)

\begin{abstract}
This is the first non-retrospective neuropsychological group study using a componential task analysis for the study of imagery. Seventy unilaterally brain damaged patients were tested on a series of tasks with the intent of isolating genuine deficits of the image generation process. The main finding is that the image generation seems to be more frequently disturbed after a left posterior damage. Suggestions are provided for further investigations.
\end{abstract}

Key Words: imagery; image generation; task analysis; cerebral localization.

\section{INTRODUCTION}

Views about hemispheric asymmetry for the generation and use of visual images have fluctuated wildly over the last decade [6]. Indeed, the earlier predominant view was that the right hemisphere is dominant for visual imagery as it was thought for visuo-spatial perceptual processes [15]. This position was first questioned in 1983 when Erlichman and Barret [3], on the basis of a review of available literature, concluded that little support was there for the hypothesis of a right hemisphere dominance. The data were equally consistent with no asymmetry at all.

However, the ability to perform an image generation task calls into play a series of operations only one of which is directly concerned with image generation per se. The reason why any firm conclusion about the lateralization of this process could not be drawn probably lay in the difficulty in distinguishing genuine problems in image generation from problems in accessory operations.

This distinction and, consequently, a fundamental improvement, came with the use of Kosslyn's [8] componential theory of visual imagery. A number of distinct operations (the retrieval of information, visual detection, inspection and recognition of the image) were identified besides the one directly concerned with image generation, that is the conversion of the information stored in long-term memory into visual, explorable, mental images. More precisely, Kosslyn distinguished different kinds of information-manipulating structures and information-bearing processes. Two kinds of structures were thought to be

†Address for correspondence and requests for reprints: Carlo Semenza M.D., Dipartimento di Psicologia Generale, Piazza Capitaniato, 3, 35139 Padova, Italy. 
involved: the long-term visual memory, which stores information about the appearances of objects, and the visual buffer, a short-term memory repository which is the medium where patterns of activation are formed (either by a visual percept or a visual mental image). The information-manipulating processes manipulate the patterns of activation in the visual buffer. The 'generation process' creates the image in the visual buffer from information stored in long-term visual memory, the 'inspection process' converts the patterns of activation in the visual buffer into organized percepts; finally, there are processes that transform the image.

The following components were added by Farah [4] to Kosslyn's original model (see Fig. 1): (a) a describe component which permits to describe the contents of the visual buffer (visual to verbal translation), (b) a copy component which is required when the contents of the visual buffer must be reconstructed (visual to motor translation), (c) a match component which is involved when the contents of the visual buffer must be compared with the contents of long-term visual memory to recognize objects, and (d) a sensory process that encodes visually presented stimuli and detects activation in the visual buffer. All the cognitive components provided by the model are supposed to be involved either in visual perception and in visual imagery, except for the generation process (the only one specific to the visual imagery).

On the basis of this model, Farah carried out a componential analysis of the imagery deficits reported in the literature to find a way to infer, from the descriptions of patients' performances in various tasks, which component of the visuo-imagery system must have been damaged. For this purpose, Farah provided a task analysis of the imagery system, that is an analysis of the cognitive components considered necessary to perform all the tasks that were felt to be relevant to imagery processes. Thus she selected and analyzed six kinds of tasks, some of which are considered to require the generation of visual images (question-answering, description from memory, drawing without model) and some considered relevant to exclude that patients' defective performance in imagery tasks can be due to a damage to other components of the system (visual detection, copying and recognition). The patterns of deficits and preserved abilities reported in neuropsychological literature proved to be instrumental in supporting empirically Farah's theoretical task analysis. While complex imagery tasks seem to be performed with the contribution of both hemispheres [see also 11], Farah's analysis showed a trend across cases suggesting a left hemisphere specialization for the image generation process.

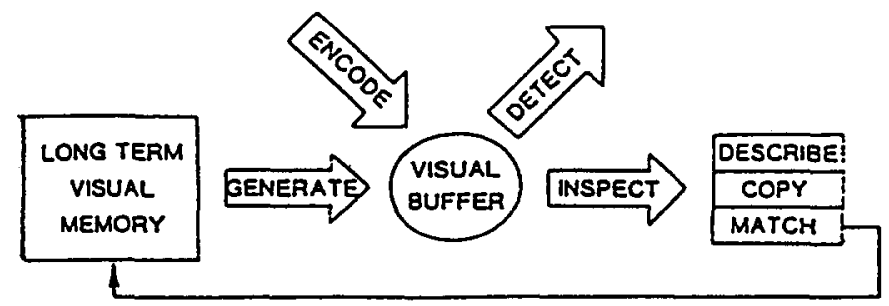

Fig. 1. Kosslyn model's modified by Farah. 
The theoretical validity of the componential approach in now universally accepted. According to later reviewers [14, 16], however, scanty descriptions and lack of critical information in earlier, retrospectively assessed, case reports do not allow any secure conclusion on lateralization. On the other hand, several contemporary group studies, while aimed at investigating imagery abilities, did not directly profit from Kosslyn's [8] and Farah's [4] methodology; indeed, they were not designed in a way that allows a precise distinction between the generation of visual representations and other processes that operate on these representations, but that may well be neurologically endowed elsewhere in the brain. Thus, as with the single case literature, recent reviews $[6,14,16]$ agree that the evidence collected for the lateralization of the image generation through group studies is at best equivocal. (The reader is referred to these reviews and other papers in the present issue for details.)

The present study intends to investigate the problem of lateralization of the image generation process by strictly following Kosslyn's [8] model and Farah's [4] indications. An adequate number of unilaterally brain-damaged patients will be tested: subjects with a genuine deficit of image generation will be singled out with the same procedure used by Farah [4] in her previous investigation, with the obvious advantage that all cases will be tested the same way and all relevant information will be available for each case.

\section{MATERIALS AND METHODS}

\section{Experimental tasks}

A battery of nine tasks was built with the aim of isolating deficits that could not be explained otherwise than with a disturbance in the process of generating images. Six of them (Visual detection, Question-answering, Description from memory, Drawing without model, Recognition, Copy) were the same sort of tasks considered by Farah [4]; the remaining three (Map drawing, Matching, Functional definition) have been added for the present study. The following is a detailed description of all the tasks. Instructions were repeated until patients showed full understanding. No time limits were required. Errors due to poor articulation in speech tasks were not counted. Each task employed will be now described in detail, in order of administration. This order was established to minimize the possibility that performance on one test would have influenced performance on following tests (e.g. 'copy' was always given after 'drawing without model' or 'description from memory'). In fact several items appeared in many tasks in order to better match the various tasks.

Visual detection. This task, derived from Humphreys and Riddock [7], was aimed at verifying the ability to detect a target stimulus out of an array of visually presented stimuli; following Farah's task analysis, its presence is necessary to exclude perceptual deficits (that is, deficits to the encode or to the detect component provided by the model). Detection was requested in three different conditions, consisting of six items each: (a) simple target, homogeneous distractors; (b) complex target, homogeneous distractors; (c) complex target, heterogeneous distractors (an example of the stimulus material used is reported in Fig. 2). Scoring assigned one point for each correct detection.

Question-answering. Subjects were required to answer 'yes or no' questions about the visual appearance of familiar objects (five items) and of famous people (five items). Examples of questions are: 'Does the President of the Italian Republic have a moustache"? Each successful answer was scored with one point. This task is considered a classical one to assess subjects' visual imagery; to correctly perform it, in terms of task analysis, one must in fact generate visual mental images into the visual buffer from information stored in long term visual memory. This task, however, may not be so sensitive; in fact, the typical questions constituting the task often appeal to knowledge that could be propositionally stored ('Was Marylin Monroe blonde?'); furthermore, questions in the yes/no form may not be the right ones if one wants to assess explicit knowledge. The possibility that a propositional strategy might be applied is reasonably lower employing a task like the following one which requires the description from memory of the visual appearances of objects.

Description from memory. Subjects were required to provide, without a visible model, a description of the visual features of 20 common objects (key, anchor, telephone, guitar, compass, bulb, padlock, hammer, scythe, thermometer, comb, scissors, pipe, glasses, typewriter, stop sign, car, house, boat, pen). A satisfying answer was considered one which gave the visual feature necessary to a listener for identifying the object or the figure. Two independent judges were requested to rate the answers as satisfying if (no matter how awkward the description) 


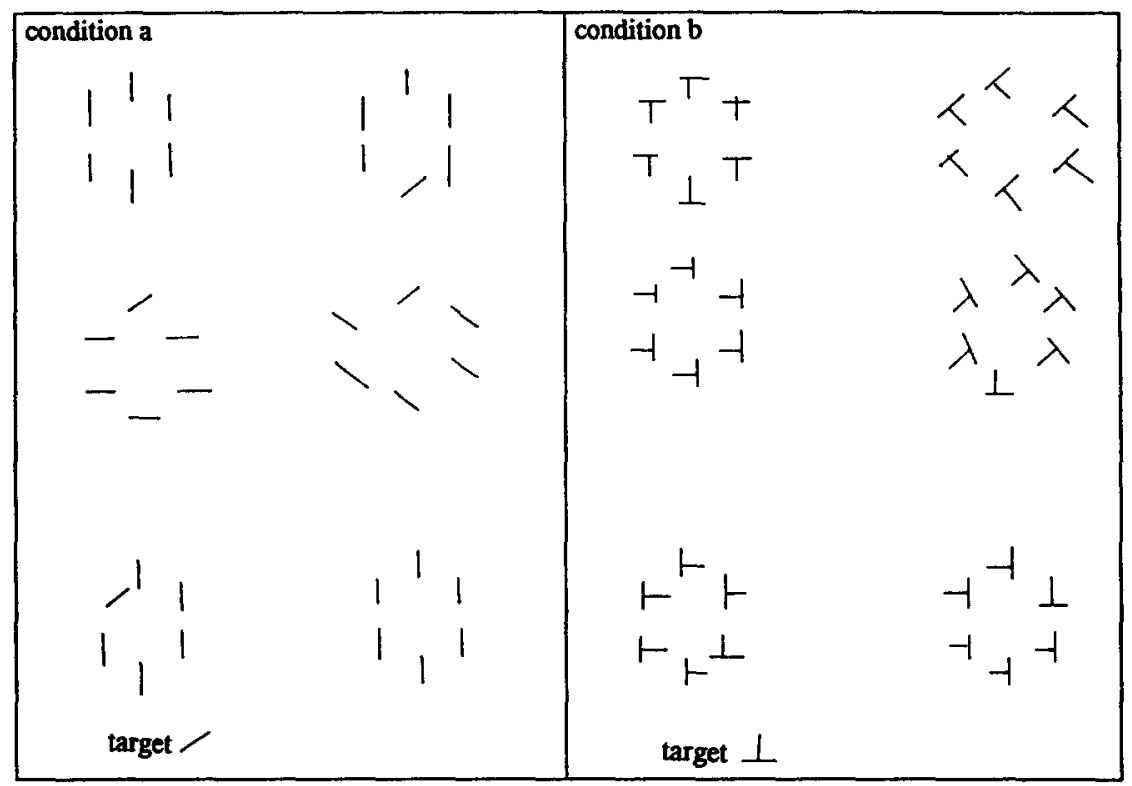

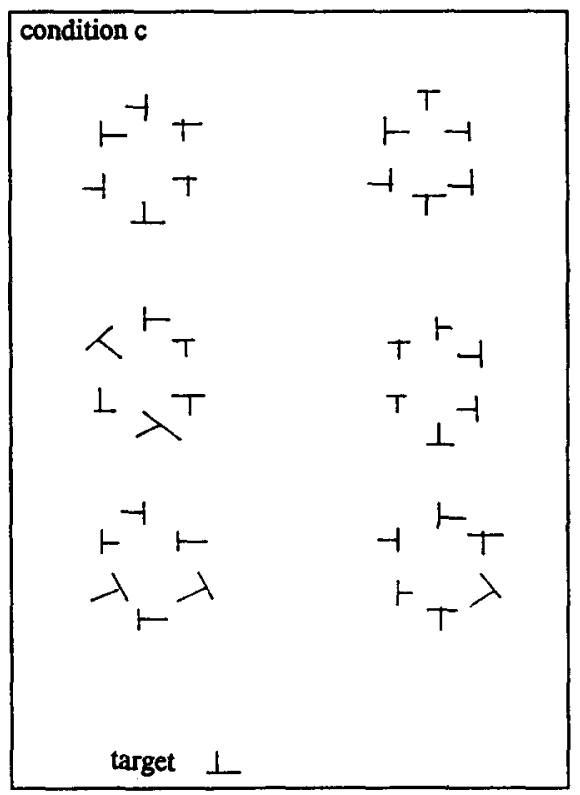

Fig. 2. Visual search task.

enough information about the structural features had been provided to enable them to guess what the object or the figure was: no wrong elements accidentally provided in the description were hidden from the judges.

Drawing without model. This task represents, together with the question-answering and the description from memory, one of the most employed tests to assess an image generation deficit. In fact, to correctly perform it, subjects have to generate visual mental images from information stored in the long term visual memory. For the present study, subjects were asked to draw, one at a time, without a model, 20 pictures of common objects (the 
same employed for the previous task). The assessment of the quality of the drawing was again asked from two independent judges (an example of the line-drawings is shown in Fig. 3). Scoring assigned one point to each correct drawing.

Map drawing. This task appears in the present study as a special case of drawing without model. It also is a task employed occasionally to assess imagery abilities. Subjects were requested to draw from memory five maps of familiar places (the hospital ward, the entire hospital, the itinerary from home to downtown, the itinerary from home to station, the home neighbourhood). Success on this task was assessed by the judges via checking the drawings with real maps and considering them as satisfactory (worth one point) if they contained main landmarks and principal directions were respected.

Recognition. Subjects were shown the drawings of common objects (same as in the other tests), one at a time, and were requested to identify them. All possible ways of identification were equally accepted and given one score point for each drawing: naming, description of feature, mime of use or matching at multiple choice with written names.

Matching. Subjects were asked to identify the correct representation of a named object within an array of four pictures (see Fig. 4). The objects were those used in the previous tasks. Distractors were either pictures of other objects with visually similar structure or representations of the same object that were less plausible or incomplete. Each correct matching was scored as one point. This task has been devised to check the integrity of the long term visual memory, more strictly, it is assumed, than via a classical recognition task. There is, in fact, the possibility that one could identify an object from only one significant peculiar detail; this does not necessarily mean that the representation of the object is completely preserved in long term visual memory. By contrast, to correctly perform the matching task, all visual features of the object, together with their spatial relations, must be preserved.

Copy. In this task subjects were requested to copy 20 line-drawings of common objects (same as in previous tasks) and 10 of complex geometrical figures (taken from Benton [1] or the Bender Gestalt Test) which they received one at time. An acceptable drawing (scored with one point) was one in which two independent judges agreed, without the model, that the target was recognizable and that main elements were properly placed in space in relation to each other. Every other less successful attempt received a score of zero.

Functional definition. In this task, subjects had to give a description of 20 names of common objects (same as in the other task) spoken by the examiner. A satisfying answer (scored with one point) was considered one which included the main functional attributes of the objects and the superordinate category. For example: a hammer is an instrument that is used to hit nails with. As it is said above this task is meant to make sure that the patient really understands the object $s /$ he is required to draw or describe from memory.

\section{Criteria for establishing an image generation deficit}

In order to infer a damage to the generation component a pattern of dissociations has to be shown such that the integrity of all processes of the imagery system but the image generation itself would be convincingly demonstrated. Again along the lines indicated by Farah it is thought that a patient could be considered as affected by a genuine defect in generating images if, vis-à- $v i s$ the battery administered in this study, $s /$ he could perform normally on the following control tasks:

Visual search. In order to rule out a perception deficit which could compromise the performance in drawing and in tasks where visual recognition is required; Recognition, in order to rule out a deficit in the long term visual memory; as noted above, however, doing well on recognition alone does not guarantee that long term visual memory is entirely preserved; Matching was designed therefore to make sure that the recognition task could not be overcome on the basis of partial information (distractors that are configurationally entirely different from the

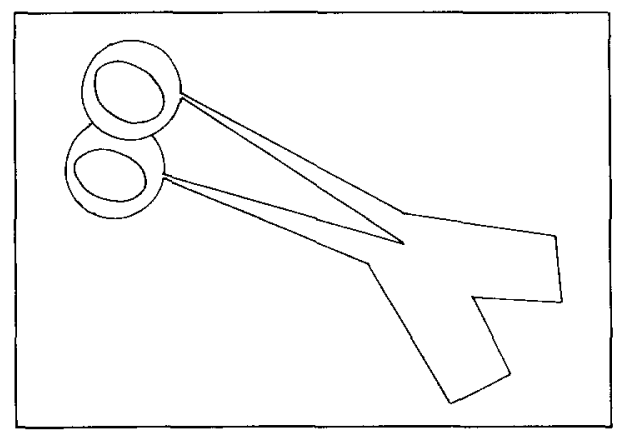

Fig. 3. Example of drawing of scissors without model from a patient with imagery generation disorders. 


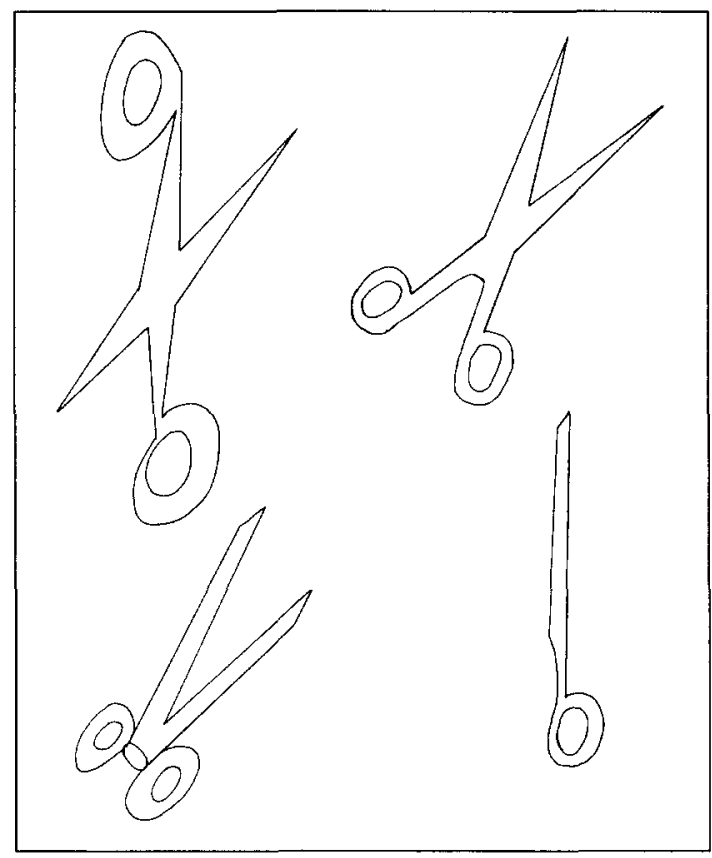

Fig. 4. Example of a stimulus material used in the matching task.

target, could, in the recognition tasks, be ruled out much more easily - see for similar problems Bishop and Byng [2]; Copy, to exclude the influence of praxic-constructional difficulties; Functional description, to exclude a wider semantic disturbance (of the kind described, for instance, by Warrington and Shallice [17]).

On the other hand, the subject should perform poorly on the critical tasks: Drawing without model (objects, figures and Map drawing), Question-answering or, at least, Description from memory.

This pattern of preserved (in control tasks) and impaired (in critical tasks) performance is therefore crucial in the present study in order to classify each single patient as affected in the image generation process itself.

\section{Subjects}

The experimental group has been selected from 82 subjects with a unilateral lesion who were consecutively admitted, over a period of about one year, to the Neurosurgery Department of the Ospedale Civile of Novara. Because of the nature of the task (which implied some cultural demands) and in order to minimize any possible effect due to a generalized memory impairment, criteria for inclusion were: age not older than 50, educational level of no less than 5 years and a health condition allowing the patients to undergo a preliminary testing session. This was conducted via the Wechsler Bellevue Intelligence Scale, the Raven Coloured Progressive Matrices and the Language examination from the Bisiach, Cappa and Vallar battery. Twelve patients (seven with a left hemispheric lesion and five with right hemispheric lesion) out of 82 were discarded after preliminary testing because their poor performance would have compromised the reliability of their answers to all the experimental tasks. Severe aphasia caused dropping of the seven left hemisphere patients while the five right hemisphere patients were discarded for either unstable emotional behaviour or unreliable level of attention.

This selection process brought to an experimental group of 70 patients, 42 males and 28 females. Their mean age was 39, ranging from 25 to 50 years, with an average educational level of 9 (5-13) years. All of them were right-handed with no first degree relatives known to be left-handed. 35 patients had a left hemispheric lesion (eight anterior and 27 posterior) and 35 had a right hemispheric lesion (12 anterior and 23 posterior).

The aetiology of the lesion was tumoral in all cases except in five vascular patients in the left hemisphere group and eight patients (seven vascular and one traumatic) in the right hemisphere group. Tumoral patients who were observed in the post-operative stage (12 in left hemisphere group and 13 in the right hemisphere group) were tested at least 2 weeks after surgery and when signs of the oedema had disappeared. Malignant tumours affected 22 out of the 32 tumoral patients who where, instead, tested before surgery. Their proportion was about the same in the four groups of patients made along the left/right and anterior/posterior dimensions.

The localization of the lesion has been made on the basis of CT scan images; this unfortunately, cannot fully prevent the inclusion in the study of patients with bilateral lesions, although any suspicious case was discarded. 
Attribution to anterior (i.e. fully frontal) or posterior region has been made according to where the bulk of the lesion appeared to be with respect to the Rolandic fissure. Control subjects were 50 non-neurological patients matched for age and educational level.

\section{RESULTS}

This study, being the first to investigate a population with this peculiar battery, takes the burden of facing the problem of scoring for some of the tasks intended to measure the imagery ability. The nature of most of such tasks makes scoring on imagery batteries very difficult. Neuropsychological works often have this problem: for instance 'severity' ratings on aphasia or even the assessing of constructional apraxia are just some of the examples that share a certain unavoidable degree of subjectivity on the scorer's part. Part of the problem was solved having independent judges agreeing on the acceptability of responses. This, however, may not be entirely satisfactory. It was decided therefore to adopt very conservative criteria to judge a performance in a given task as truly differing from normality. Mean scores, expressed as percentage correct, for each task in the control group are shown in Table 1.

A minimum of 3 S.D. below the mean performance of normal patients (in any case lower than every single score from the control group) was set as a cut-off point for considering the performance as pathological. In the tasks where every normal subject performed at ceiling the cut-off point was established below $80 \%$ of the maximum score.

Given the criteria outlined above, 19 subjects showed the right pattern of dissociations to be singled out as having a demonstrable selective deficit of the visual image generation process. In fact they failed in the critical tasks (drawing without model, map drawing, description from memory and some also in question-answering, which turned out to be a very easy task in the form we provided), but were unimpaired in visual search, recognition, matching, copy and functional description. As already seen in the methods section, a good performance in the latter group of tasks guarantees that a bad performance in the critical tasks is not due to damage to processes other than the image generation process itself (for instance patients who cannot draw because of apraxic problems or who cannot describe because of faulty long-term memory). Fifteen of the 19 eligible patients had left hemisphere lesions, only one of which appeared to be entirely delimited within the anterior region. The remaining four patients had a posterior lesion in the right hemisphere. None of these patients ever explicitly complained of imagery loss. An additional subset of patients (seven subjects, four with a left-sided lesion - one anterior and three posterior-and three with a right-sided lesion) would also have been classified as selectively defective in the generation process, but for their failure in the matching task. Only four patients who were affected by a malignant tumour and were tested preoperatively (i.e. the patients for which localization is more problematic) appeared in the 19 critical patients group: three had a left posterior lesion and the other was the only patient with a left anterior lesion. Despite this, the preponderance of tumoral patients may indeed be seen as a problem for this or any other study on localization of functions. A new investigation, currently being conducted at the Ospedale Maggiore of Trieste (Semenza, Pelizzon, Zadini and Fossella, in preparation) with a longer battery, reveals however that the same distribution of image generation disturbances holds with vascular patients. 


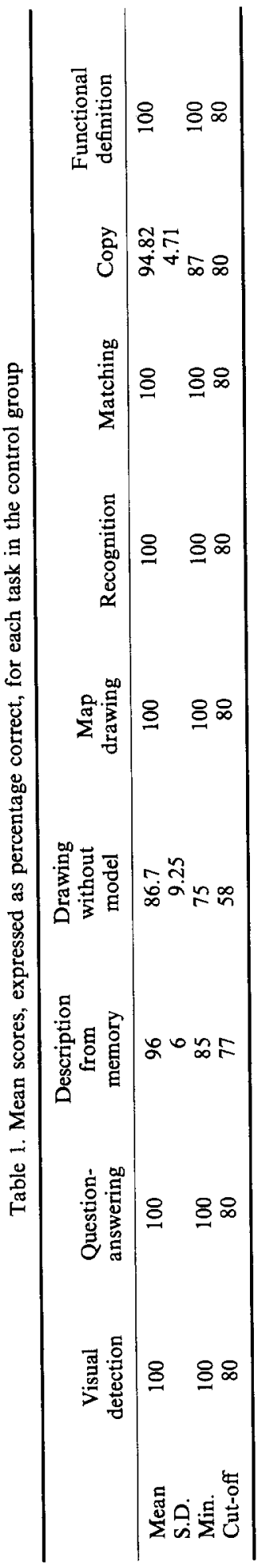




\section{DISCUSSION}

The outcome of the present investigation is rather straightforward. A functionally isolable deficit in the image generation process may be found, in the overwhelming proportion of the cases, after a posterior left hemisphere lesion. This finding confirms and clearly supports Farah's [4] conclusions from backward examination of the literature. Basically the same method of assessing patients according to their patterns of deficit and preserved abilities was adopted. Indeed this study may be considered a collection of single case studies where performance was never averaged over cases.

It is important to stress the fact that the conclusive figures are based on very conservative inclusion criteria. As already reported, an additional number of patients would also have been classified as selectively defective in the generation process but for their failure in the 'matching' task. Their problem is indeed better understood as a subtle deficit on long-term memory. Their stored visual representation, while retaining most basic features, would be lacking the precision over details that would have allowed a flawless performance in the 'matching' task. This said, it must be observed that many other patients participating in the study may have had problems in image generation. Concomitant symptoms make their cases not transparent enough to allow conclusions about whether they genuinely suffered in image generation. In the same line it must be pointed out that the criteria for participating in the present study were very strict about general awareness, age (an unusually low threshold is adopted), and education (though less strict) in order to avoid problems in the comprehension of task requirements. There is no reason, of course, to think that older, less-educated and less-alert patients would not be affected by image generation deficits.

All this brings to the conclusion that genuine image generation deficits are rather common, a conclusion that somehow contrasts with the lack of reliable clinical data lamented by recent critical reviewers of available literature $[14,16]$. Indeed the battery of tasks built for this study may turn out to be sensitive to very mild imagery deficit that would not be so evident without specific clinical detection (remember that none of the patients spontaneously complained of imagery loss). Cases so far retrospectively judged as having an image generation deficit may well have been very severe and thus easier to be spotted.

The fact that a few patients (five out of 19) were found who seemingly have a genuine deficit in the generation process, and yet a lesion outside the left posterior areas may be interpreted in various ways. An artefact in patient selection is the most obvious and perhaps less interesting cause for this finding: additional lesions or areas of dysmethabolism in the left posterior region may have escaped the CT scan scrutiny. The same investigation via more sensitive neuroimaging techniques is obviously called for. A further possibility is that, indeed, as hypothesized by Sergent [14] or Kosslyn [9] both hemispheres simultaneously and conjointly contribute to the image generation process. In this case, however, vis-à-vis the results discussed here, the process seems to have a larger neurological implementation in the posterior left hemisphere. The converse problem is why some patients with a posterior left hemisphere lesion do not have an imagery deficit. The left postrolandic/suprasylvian area, however, is huge and anatomically and functionally dishomogeneous: there is no wonder that some lesions to this area would leave the imagery abilities intact. However the data collected in the present CT scan investigation does not provide indications helping to circumscribe the localization of the function any further. 
Finally individual variability might be considered. Little is known or can presently be speculated about different strategies in determining image generation or about the role of different expertise (see [14] for suggestions on this last point). The combination of these sorts of factors with a possible variation in the anatomical organization is a classic difficulty in neuropsychology [5, 10-12] specially when broadly specified issues like imagery are dealt with.

The realm in which the imagery deficit has been judged with this battery concerns a limited number of items. This is a choice dictated by the necessity of keeping the administration of the battery within a reasonable amount of time given the post/pre operatory condition of most patients. Also, as it has already been mentioned, the same items were used over different tasks with the intent to control as much as possible for item difficulty. Although all highly imaginable the items did not belong to the same category and are encountered in real life in very different contexts. This provides same guarantee that what is explored is not a specific domain but a vast realm of knowledge. However living things were not included in this study and must be taken into account in next investigations.

In summary this is the first non-retrospective neuropsychological group study using a componential task analysis for the study of imagery. The main finding is that the image generation process seems to be more frequently disturbed after a left posterior damage. It may well be the case that, while the tasks considered for this study are really processed in the left hemisphere, other image tasks have an opposite hemispheric lateralization. Whether or not, therefore, the left posterior area or a portion of it would ultimately turn out to be the only critical one for the image generation process would need further investigation via (1) better neuroimaging techniques and (2) more extensive and more comprehensive tests. More importantly, however, an even more detailed theory and task analysis would be the factors expected to lead to real progress. The present investigation must, by all means, be considered preliminary and exploratory of possible problems never really faced before. Yet it was a necessary step.

\section{REFERENCES}

1. Benton, A. L. Constructional apraxia and the minor hemisphere. Confinia Neurologica 29, 1-16, 1967.

2. Bishop, E. S. and Byng, S. Assessing on comprehension: methodological considerations and a new clinical test. Cognit. Neuropsychol. 1, 223-243, 1984.

3. Erlichman, H. and Barret, J. Right hemispheric specialization for mental imagery: A review of the evidence. Brain Cognit. 2, 55-76, 1983.

4. Farah, M. J. The neurological basis of mental imagery: A componential analysis. Cognition 18, 245-272, 1984.

5. Farah, M. J. Neuropsychological inference with an interactive brain: A critique of the "locality assumption". Behav. Brain Sci. 17, 43-61, 1994.

6. Hellige, J. B. Hemispheric Asymmetry. Harvard University Press, Cambridge, Mass., 1993.

7. Humphreys, G. W. and Riddock, M. J. (Editors) The fractionation of visual agnosia. In Visual Object Processing. A Cognitive Neuropsychological Approach. Lawrence Eribaum, London, 1987.

8. Kosslyn, S. M. Image and Mind. Harvard University Press, Cambridge, Mass., 1980.

9. Kosslyn, S. M. Seeing and imagining in the two cerebral hemispheres: A computational approach. Psychol. Rev. 94, 148-175, 1987.

10. Semenza, C., Bisiacchi, P. S. and Rosenthal, V. A function for cognitive neuropsychology. In Perspectives on Cognitive Neuropsychology, G. Denes, C. Semenza and P. S. Bisiacchi (Editors). Lawrence Erlbaum, London, 1988.

11. Semenza, C. Locus-pocus. (Which and whose locality assumption?) A commentary on Martha Farah: Neuropsychological inference with an interactive brain: A critique of the "locality assumption". Behav. Brain Sci. 17, 80, 1994. 
12. Semenza, C. Methodological issues. In The Blackwell Dictionary of Neuropsychology, G. Beaumont, P. Kenealy and M. Rogers (Editors). Basil Blackwell, London, 1995.

13. Sergent, J. Judgements of relative position and distance on representations of spatial relations. $J$. exp. Psychol.: Hum. Percept. Perform. 91, 762-780, 1991.

14. Sergent, J. The neuropsychology of visual image generation. Brain Cognit. 13, 98-129, 1990.

15. Springer, S. and Deutsch, G. Left Brain, Right Brain. Freeman, San Francisco, California, 1981.

16. Troiano, L. and Grossi, D. A critical review of mental imagery defects. Brain Cognit. 24, 213-243, 1994.

17. Warrington, E. K. and Shallice, T. Category-specific semantic impairments. Brain 107, 829-854, 1984. 